TITLE:

\title{
Prenylation of aromatic compounds, a key diversification of plant secondary metabolites
}

$\operatorname{AUTHOR}(S)$ :

Yazaki, Kazufumi; Sasaki, Kanako; Tsurumaru, Yusuke

\section{CITATION:}

Yazaki, Kazufumi ... [et al]. Prenylation of aromatic compounds, a key diversification of plant secondary metabolites. Phytochemistry 2009, 70(15-16): 1739-1745

ISSUE DATE:

2009-10

URL:

http://hdl.handle.net/2433/87735

\section{RIGHT:}

c 2009 Elsevier Ltd. All rights reserved.; This is not the published version. Please cite only the published version.; この論文は出版社版でありませ ん。引用の際には出版社版をご確認ご利用ください。 
Prenylation of aromatic compounds, a key diversification of plant secondary metabolites

Kazufumi Yazaki*, Kanako Sasaki, and Yusuke Tsurumaru

Laboratory of Plant Gene Expression, Research Institute for Sustainable Humanosphere, Kyoto University, Gokasho, Uji 611-0011 Japan

Review (Special Issue on Evolution of Metabolic Diversity)

* Corresponding author. E-mail address: yazaki@rish.kyoto-u.ac.jp, Tel: +81-774-38-3617; Fax: +81-774-38-3623

Keywords: Dimethylallyl diphosphate; Flavonoid; Membrane-bound enzyme ; Metabolic diversity; Polyphenol; Prenyltransferase. 
Table of Contents

1. Introduction

2. Structures and biological activities of prenylated polyphenols

3. Biochemical research on prenyltransferases for polyphenols

4. Isolation of flavonoid-specific prenyltransferase cDNAs from plants

5. Production of prenylated flavonoids in heterologous systems

6. Concluding remarks

Acknowledgments

References 


\section{Abstract}

Prenylation plays a major role in the diversification of aromatic natural products, such as phenylpropanoids, flavonoids, and coumarins. This biosynthetic reaction represents the crucial coupling process of the shikimate or polyketide pathway providing an aromatic moiety and the isoprenoid pathway derived from the mevalonate or MEP (methyl erythritol phosphate) pathways, which provides the prenyl (isoprenoid) chain. In particular, prenylation contributes strongly to the diversification of flavonoids, due to differences in the prenylation position on the aromatic ring, various lengths of prenyl chain, and further modifications of the prenyl moiety, e.g. cyclization and hydroxylation, resulting in the occurrence of ca. 1,000 prenylated flavonoids in plants. Many prenylated flavonoids have been identified as active components in medicinal plants with biological activities, such as anti-cancer, anti-androgen, anti-leishmania, and anti-nitric oxide production. Due to their beneficial effects on human health, prenylated flavonoids are of particular interest as lead compounds for producing new drugs and functional foods. However, the gene coding for prenyltransferases that catalyze the key step of flavonoid prenylation have remained unidentified for more than three decades, because of the membrane-bound nature of these enzymes.

Recently, we have succeeded in identifying the first prenyltransferase gene SfN8DT-1 from Sophora flavescens, which is responsible for the prenylation of the flavonoid naringenin at the 8-position, and is specific for flavanones and dimethylallyl diphosphate (DMAPP) as substrates. Phylogenetic analysis showed that SfN8DT-1 has the same evolutionary origin as prenyltransferases for vitamin $\mathrm{E}$ and plastoquinone. A prenyltransferase GmG4DT from soybean, which is involved in the formation of glyceollin, was also identified recently. This enzyme was specific for pterocarpan as its aromatic substrate, and (-)-glycinol was the native substrate yielding the direct precursor of glyceollin I. These enzymes are localized to plastids and the prenyl chain is derived from the MEP pathway. Further relevant genes involved in the prenylation of other types of flavonoid are expected to be cloned by utilizing the sequence information provided by the above studies. 


\section{Introduction}

Polyphenols are common secondary metabolites in all plant species and are widely known as natural antioxidants (D'Archivio et al., 2007). Recently, more divergent biological activities, e.g. prevention of high blood pressure and of hardening of veins, anti-aging, anti-bacterial, and anti-tumor activities, have been reported for various polyphenolic compounds as valuable natural products that are beneficial for human health. In the market, many kinds of functional foods and supplements containing these polyphenols are widely sold. Polyphenolic compounds exist in fresh plant cells, in most cases, as derivative forms, such as methyl ethers, glycosides, and other decorations are also often observed. It is worth noting that some of these derivatives exhibit much higher biological activities than their mother compounds without derivatization or decoration, and polyphenol derivatives showing high biological activities have been isolated from various medicinal plants. A large variety of biological activities have been reported particularly in polyphenols having prenyl residues that consists of isoprene units with 5 carbon atoms, and these plant products provide rich resources for natural medicines (Botta et al., 2005). Thus far, more than 1,000 prenylated polyphenols have been isolated from plants, which has drawn substantial attention in the field of applied sciences, such as food industries, breweries, cosmetic companies, etc. This review provides an overview of prenylated polyphenols showing various biological activities and introduces recent discoveries of plant genes encoding flavonoid-specific prenyltransferases; key enzymes in biosyntheses of those prenylated polyphenols.

\section{Structures and biological activities of prenylated polyphenols}

Plant polyphenols are classified into several groups according to the basic ring system, e.g. phenylpropanoids, flavonoids, coumarins, phloroglucinols, and xanthones. There seems to be a chemotaxonomical tendency in the occurrence of some polyphenols, e.g., xanthone derivatives mostly occur in Guttiferae (Clusiaceae) (Pinto et al., 2005), and ca. 90\% isoflavonoids are derived from Leguminosae (Fabaceae) (Dixon, 2009), but other groups of polyphenols are widely distributed in the plant kingdom. The occurrence of prenylated polyphenols are rather limited in several plant families. Representatives include Leguminosae (Fabaceae), Moraceae, Cannabaceae, Guttiferae (Clusiaceae), Umbelliferae and Rutaceae (Park et al., 2003; Stevens et al., 2000; Wu et al., 1998; Aoki et al., 2008; Ribeiro et al., 2008.), whereas some other plant families like 
Euphorbiaceae and Compositae (Asteraceae) also comprise plant species that contain prenylated polyphenols (Kumazawa et al., 2007, 2003). Plants containing these compounds have often been utilized as medicinal plants in many countries, for example, licorice (Leguminosae) is used as an anti-inflammatory in Chinese traditional medicine (Shin et al., 2008), Calophyllum inophyllum (Guttiferae) is used against bronchitis and diarrhea in Latin America (Mesia-Vela et al., 2001), and osage orange (Maclura pomifera) is used for cancer treatment (Mahmoud, 1981).

A wide range of biological activities has been reported for prenylated polyphenols, e.g. anti-tumor, anti-bacterial, anti-virus, anti-oxidant, anti-tyrosinase, estrogenic, inhibition of sulfotransferase, anti-nitric oxide production, and inhibition of phospholipase (Miranda et al., 2000; Appendino et al., 2008; Lee et al., 2009; Kapche et al., 2009; Kim et al., 2003; Dong et al., 2007; Mesia-Vela et al., 2001; Lee et al., 2005; Oh et al., 2005). A representative of the prenylated flavonoids, 8-dimethylallyl naringenin, has been identified in some leguminosaeous plants and is recognized as a strong phytoestrogen leading to its potential usage for the prevention of osteoporosis and for the enhancement of collagen synthesis in the skin (Tielens et al., 2008). Xanthohumol is another example of important prenylated flavonoids for its divergent biological activities, such as estrogenic, anti-oxidant, anti-tumor and so on. This compound is the main component (80 -90 \% of total flavonoids) in hops (Humulus lupulus L., Cannabaceae), which are used to add bitterness and flavor to beer (Stevens and Page, 2004). Also found in hops, humulone and lupulone, phloroglucinol derivatives known as the bitter principle of beer, exhibits anti-tumor activity via the inhibition of cyclooxygenase-2 expression, which is mediated by a signal transduction pathway with transcriptional regulators NF-kB and AP-1 (Lee et al., 2007). As an example of a xanthone derivative, prenylated xanthone (rubraxanthone) identified in Garcinia dioica (Guttiferae) shows antithrombotic, anti-allergic, and anti-inflammatory activities via suppression of the binding of platelet activation factor to its receptor (Iinuma et al., 1996; Jantan et al., 2002). The enhancement of drug effects has been reported in prenylated furanocoumarin of grapefruit, which is caused by the inhibition of enzymes of drug metabolism in the human intestine (Row et al., 2006).

Even a single prenylated compound may show multiple effects, e.g., kurarinone (a prenylated flavanone) isolated from Sophora flavescens (Leguminosae) exhibits estrogenic, anti-tyrosinase, anti-glycosidase, and anti-lipoxygenase activities (De 
Naeyer et al., 2004; Kim et al., 2003, 2006; Chi et al., 2001; Yamahara et al., 1990) (Fig. 1.). It is noteworthy that the prenyl moiety often plays a crucial role in these divergent biological activities in many of these compounds (Row et al., 2006). This suggests, in turn, the addition of a prenyl residue to polyphenol skeletons may contribute to the enhancement of the biological activities of polyphenolic compounds.

For plants, prenylated polyphenols function as protectants against pathogenic microorganisms and herbivores (Moesta et al., 1983; Robbins et al., 1985), or they may act against abiotic environmental stresses like oxidative stress as they show strong anti-oxidant activity (Kumazawa et al., 2007). Humans utilize these active compounds for multiple purposes in different fields, such as pharmacognosy, food chemistry, and agriculture, according to their divergent chemical and biological activities. Despite these attractive features, the stable supply of prenylated polyphenols is, however, at present hardly achieved due to their low content in natural sources, their existence as a complex mixture in plant extracts, occurrence in rare plant species, and/or the lack of economical production systems.

\section{Biochemical research on prenyltransferases for polyphenols}

The enzymatic reaction step catalyzed by polyphenol prenyltransferase represents the crucial coupling reaction of two major metabolic pathways, i.e., aromatic compounds biosynthesis routs such as the shikimate and acetate / malonate (polyketide) pathway, and isoprenoid biosynthesis routs such as mevalonate and MEP pathway. Prenyltransferases for polyphenols is thus the key biosynthetic enzymes of these compounds in plants (Fig. 2.), and they have been studied intensively world-wide, especially in Germany, Canada, and the US for more than three decades. Glyceollins, for instance, are widely known phytoalexins produced by soybean, and their biosynthetic enzymes were actively studied especially from 1970s to 1990s (Ebel and Grisebach, 1988). Glyceollins are derivatives of the pterocarpan designated as glycinol, to which a dimethylallyl residue is introduced on an aromatic ring. The prenyltransferase activity of glycinol was reported in the 1970s (Zahringer et al., 1979). Another prenyltransferase activity involved in the prenylation of coumarin was also studied in rutaceous plants at a biochemical level in detail (Ellis and Brown, 1974; Hamerski et al., 1990). Both enzymes were reported to be membrane-bound enzymes and localized to the plastid in plant cells (Table 1). In another example, the prenyl 
moiety of an anti-bacterial flavonoid, glabrol, of licorice (Glyzzirhisa spp.) was proved to be derived from MEP pathway, suggesting that the prenyltransferase involved is also localized to the plastid (Asada et al., 2000).

Soluble type prenyltransferases were also reported in some plant species. The prenyltransferase responsible for the biosynthesis of hallucinogen, one of the cannabinoids, in hemp is olivetolate geranyltransferase, whose activity was detected in a soluble fraction (Fellermeier and Zenk, 1998). The prenyltransferase activity involved in humulone biosynthesis was also reported to be localized in a soluble fraction (Zuurbier et al., 1998), whereas the prenyl moiety was shown to be derived from the MEP pathway. Thus, it is in general believed that the prenyl chain of polyphenols is derived from the MEP pathway regardless the type of enzymes and aromatic prenyl acceptor molecules (Table 1).

In contrast to those biochemical studies, there has been neither an example of the purification of polyphenol prenyltransferases to homogeneity nor the isolation of genes coding for these prenyltransferases from plants; therefore, it has long been a mystery what kind of proteins catalyze these prenylation reactions in plants. Only in fungi and bacteria have soluble-type prenyltransferases been reported, which prenylated indole derivatives and polyketides, respectively (Kuzuyama et al., 2005; Kumano et al., 2008; Steffan et al., 2009; Li, 2009). For more detail of these prenyltransferases in fungi and bacteria, a recent review by Heide summarized new knowledge in this field (Heide, 2009). However, orthologues of these soluble-type prenyltransferases have not been found in plants thus far, and plant enzymes responsible for the prenylation of aromatic compounds have remained as a big black box in the research field of plant secondary metabolism.

\section{Isolation of flavonoid-specific prenyltransferase cDNAs from plants}

Recently, we have succeeded in cloning a cDNA encoding a prenyltransferase specific for an endogenous flavanone from cultured S. flavescens cells, which are capable of producing sophoraflavanone G, a prenylated flavonoid, in large amounts (Sasaki et al., 2007). Because no amino acid sequence information was available for flavonoid-specific prenyltransferases in plants, an EST data (ca. 12,000) was first obtained from cultured S. flavescens cells, and narrowed down candidate clones with three criteria based on the biochemical information, i.e., (1) the target protein should 
have an aspartate-rich motif as conserved among the Mg-dependent prenyltransferase family, (2) a plastid-targeting signal (transit peptide) should be detected at the N-terminus, and (3) the clone should possess at least one transmembrane alpha-helix. Out of ca. $10 \mathrm{k}$ EST sequences only 7 clones fulfilled these criteria. These candidates were expressed in a yeast strain, in which the endogenous aromatic substrate prenyltransferase gene coq2 had been disrupted to decrease the possible background (Yazaki et al., 2002). In an enzyme assay using the microsomal fraction of yeast transformants, one clone gave clear prenyltransferase activity with dimethylallyl diphosphate (DMAPP) and naringenin as substrates. Because this enzyme was specific for DMAPP as the prenyl donor and also specific for flavanone as the prenyl acceptor as well as the prenylation at position 8 of naringenin, it was designated as SfN8DT-1 (naringenin 8-dimethylallyltransferase) (Sasaki et al., 2007).

Membrane-bound prenyltransferases of plants accepting aromatic substrates are classified into two major groups, i.e., (1) p-hydroxybenzoate (PHB) prenyltransferases and (2) homogentisate (HG) prenyltransferases. The formers are located in the inner membrane of mitochondria and are involved in ubiquinone (coenzyme Q) biosynthesis (Okada et al., 2004; Ohara et al., 2006). In this group, there is one exception, which is LePGT1 involved in naphthoquinone biosynthesis. The LePGT1 polypeptide is localized to the endoplasmic reticulum and shows strict substrate specificity for geranyl diphosphate as the prenyl donor (Yazaki et al., 2002). The latters, homogentisate prenyltransferases, are responsible for the biosynthesis of vitamin $\mathrm{E}$ or plastoquinone and are localized to the plastid (Hunter and Cahoon, 2007; Maeda and DellaPenna, 2007). Both members actually catalyze similar reactions, i.e. aromatic proton substitution with a prenyl chain either on PHB or HG molecules. While the amino acid sequence similarity of these enzymes is low (10-20\% identity), their membrane topology is very similar, i.e. 7 to 9 transmembrane alpha-helices are distributed throughout the polypeptides (Ohara et al., 2009). SfN8DT-1 is also presumed to have 9 transmembrane alpha-helices (Fig. 3.) and share significant similarity with HG prenyltransferase members (ca. 50\%) (Fig. 4). A couple of paralogues of SfN8DT were also cloned from S. flavescens, SfN8DT-2 and -3, whose gene products showed different $\mathrm{Km}$ values but almost identical substrate and product specificities as SfN8DT-1 (our unpublished data). In the molecular evolution process, flavonoid prenyltransferases were derived from the HG prenyltransferase family and acquired specificity for 
flavonoids as their substrates. The phylogenetic tree depicts that enzymes accepting flavonoids as substrates form a clade on their own (Sasaki et al., 2007) .

Almost 1,000 structures of prenylated flavonoids have been elucidated to date. It is expected that other prenyltransferases from various plant families recognizing other flavonoid types or more divergent polyphenol molecules as prenyl acceptor will be identified by utilizing the sequence information of SfN8DTs. Indeed, a pterocarpan-specific prenyltransferase, which shares significant similarity (ca. 50\%) with SfN8DTs has been identified very recently in soybean and was designated as GmG4DT because it shows strong substrate specificity for DMAPP and the native pterocarpan substrate, glycinol (Akashi et al., 2009). As the first prenyltransferase responsible for glyceollin biosynthesis, this cDNA might have the potential to be applied in agricultural field. Further isolation of new prenyltransferases showing different enzymatic properties from various plant origins is further expected.

\section{Production of prenylated flavonoids in heterologous systems}

As cDNAs of prenyltransferases for flavonoids have been isolated, the establishment of production systems for prenylated flavonoids in heterologous organisms is now feasible, either by metabolic engineering in plants or biotransformation in microorganisms. Because the occurrence of prenylated flavonoids is limited in some plant families, it was first attempted to express SfN8DT-1 in a model plant that did not produce prenylated polyphenols, and it was analyzed if the heterologous host could synthesize prenylated flavonoids. The SfN8DT-1 cDNA was ectopically expressed in Arabidopsis thaliana under the control of a CaMV35S promoter, which lead somehow to unexpected results. While wild-type plants did not produce prenylated flavonoids, the transgenic Arabidopsis accumulated 8-prenylated kaempferol (des-O-methylanhydroicaritin) as the sole detectable product (Sasaki et al., 2009). Kaempferol was not accepted as the substrate of SfN8DT-1 in an in vitro assay with recombinant enzymes (Sasaki et al., 2007). When the flavonoid substrate naringenin was fed to the transgenic Arabidopsis, an appreciable amount of the direct enzymatic reaction product 8-dimethylallyl naringenin (8DN) was detected in the transgenic Arabidopsis, in which, surprisingly, a similar level of 8-prenylated kaempferol, as well as prenylated apigenin and quercetin, was also detected while these flavones were not prenylated in the in vitro assay. The reason was presumed as follows; 
because naringenin is the initial flavonoid molecule biosynthesized in vivo and this intermediate is prenylated by the ectopically expressed SfN8DT-1 in the transgenic Arabidopsis, this product was further converted into various flavonoids such as flavones and flavonols by endogenous biosynthetic enzymes of flavonoids regardless of the prenyl chain attached at the 8-position. Kaempferol is the flavonoid that is most preferentially accumulated in Arabidopsis leaves. There may be a 'default' for the product accumulation pattern depending on the plant species, and naringenin is not a preferable form as the main flavonoid accumulated in Arabidopsis and is further converted to a kaempferol derivative, which is more acceptable for accumulation in this plant species. Although the possibility that the substrate specificity was strongly altered in vivo cannot be excluded, prenylated flavone formation was in fact only observed in Arabidopsis expressing SfN8DT-1, but not in other host plants, such as tomato and Lotus japonicus (our unpublished data). The yield was calculated as being ca. $5 \mu \mathrm{g} / \mathrm{g}$ dry wt when the transgenic Arabidopsis seedlings were grown on agar plates (Sasaki et al., 2009).

More recently, yeast transformants expressing SfN8DT-1 has been applied to biotransformation with the aim of the formation of 8-dimethylallylnaringenin, which has a large application potential as a strong phytoestrogen. In this experiment, bacteria was not applicable as a host microorganism because membrane proteins are in general not accepted to be expressed heterologously in prokaryotes. At the beginning of culture, naringenin was supplied to the medium and the yeast cells were grown in SD-medium for $12 \mathrm{~h}$ (Sasaki et al., 2009). The product, 8-dimethylallylnaringenin, was solely detected in the medium, which was easily recovered by partitioning with ethyl acetate. Because the optimization of the biotransformation has not been completed, the production level upon the addition of $0.2 \mathrm{mM}$ naringenin to the medium was as low as 0.3 - $0.5 \mathrm{mg} / \mathrm{l}$ medium, but detailed optimization of production conditions may improve the production rate considerably.

\section{Concluding remarks}

The first identification of a gene coding for a plant prenyltransferase responsible for the prenylation of flavonoids enabled the discovery of many new prenyltransferase genes from many non-model plants, such as crops, fruits, and medicinal plants, where 
the sequence information of SfN8DT and GmG4DT will be useful. It is expected that in the near future genes for important prenyltransferases for other polyphenolic compounds, such as phenylpropanoids, coumarins, and phloroglucinols will be cloned. The accumulation of polypeptide sequence information of those orthologues from a wide variety of plant species will help our understanding of the essential amino acids for the specific recognition of aromatic substrates and also for the catalytic functions.

\section{Acknowledgments}

Cultured S. flavescens cells and the intact plants were provided by Dr. H. Yamamoto of Toyo University and Kyoto Botanical Garden of Takeda Chemical Industries, respectively. Soybean GmG4DT was identified in collaboration with Dr. S. Ayabe and co-workers of Nippon University.

Authors' work introduced in this review was supported in part by a Grant-in-Aid for Scientific Research (No. 21310141 to K. Y.). 
Bibliography

\section{References}

Akashi, T., Sasaki, K., Aoki, T., Ayabe, S., Yazaki, K., 2009. Molecular cloning and characterization of a cDNA for pterocarpan 4-dimethylallyltransferase catalyzing the key prenylation step in the biosynthesis of glyceollin, a soybean phytoalexin. Plant Physiol. 149, 683-693.

Aoki, N., Muko, M., Ohta, E., Ohta, S., 2008. C-Geranylated chalcones from the stems of Angelica keiskei with superoxide-scavenging activity. J. Nat. Prod. 71, 1308-1310.

Appendino, G., Gibbons, S., Giana, A., Pagani, A., Grassi, G., Stavri, M., Smith, E., Rahman, M., 2008. Antibacterial cannabinoids from Cannabis sativa: A structure-activity study. J. Nat. Prod. 71, 1427-1430.

Asada, Y., Li, W., Yoshikawa, T., 2000. Biosynthesis of the dimethylallyl moiety of glabrol in Glycyrrhiza glabra hairy root cultures via a non-mevalonate pathway. Phytochemistry 55, 323-326.

Biggs, D.R., Welle, R., Visser, F.R., Hans, G., 1987. Dimethylallylpyrophosphate: 3,9-dihydroxypterocarpan 10-dimethylallyl transferase from Phaseolus vulgaris. Identification of the reaction product and properties of the enzyme FEBS Lett. 220, 223-226.

Botta, B., Vitali, A., Menendez, P., Misiti, D., Delle, M.G., 2005. Prenylated flavonoids: Pharmacology and biotechnology. Curr. Med. Chem. 12, 713-739.

Boubakir, Z., Beuerle, T., Liu, B., Beerhues, L., 2005. The first prenylation step in hyperforin biosynthesis. Phytochemistry 66, 51-57.

Chi, Y.S., Jong, H.G., Son, K.H., Chang, H.W., Kang, S.S., Kim, H.P., 2001. Effects of naturally occurring prenylated flavonoids on enzymes metabolizing arachidonic acid: Cyclooxygenases and lipoxygenases. Biochem. Pharmacol. 62, 1185-1191.

D'Archivio, M., Filesi, C., Di Benedetto, R., Gargiulo, R., Giovannini, C., Masella, R., 2007. Polyphenols, dietary sources and bioavailability. Annali dell'Istituto superiore di sanita 43, 348-361.

De Naeyer, A., Vanden Berghe, W., Pocock, V., Milligan, S., Haegeman, G., De Keukeleire, D., 2004. Estrogenic and anticarcinogenic properties of kurarinone, a lavandulyl flavanone from the roots of Sophora flavescens. J. Nat. Prod. 67, 
1829-1832.

Dhillon, D.S., Brown, S.A., 1976. Localization, purification, and characterization of dimethylallylpyrophosphate:umbelliferone dimethylallyltransferase from Ruta graveolens. Arch. Biochem. Biophys. 177, 74-83.

Dixon, R.A., 1999. Isoflavonoids: biochemistry, molecular biology and biological functions, In: Sankawa, U., Barton, D.H.R, Nakanishi, K., Meth-Cohn, O. (Eds.), Comprehensive Natural Products Chemistry. Vol. 1, Elsevier, Oxford, pp. 773-823.

Dong, X.W., Fan, Y.J., Yu, L.J., Hu, Y.Z., 2007. Synthesis of four natural prenylflavonoids and their estrogen-like activities. Arch. Pharm. 340, 372-376.

Ebel, J., Grisebach, H., 1988. Defense strategies of soybean against the fungus Phytophthora megasperma f. sp. glycinea: a molecular analysis. Trends Biochem. Sci. 13, 23-27.

Ellis, B.E., Brown, S.A., 1974. Isolation of dimethylallylpyrophosphate - umbelliferone dimethylallyltransferase from Ruta graveolens. Can. J. Biochem. 52, 734-738.

Fellermeier, M., Zenk, M.H., 1998. Prenylation of olivetolate by a hemp transferase yields cannabigerolic acid, the precursor of tetrahydrocannabinol. FEBS Lett. 427, 283-285.

Hamerski, D., Schmitt, D., Matern, U., 1990. Induction of two prenyltransferases for the accumulation of coumarin phytoalexins in elicitor-treated Ammi majus cell suspension cultures. Phytochemistry 29, 1131-1135.

Heide, L., 2009. Prenyl transfer to aromatic substrates: genetics and enzymology. Curr. Opin. Chem. Biol. 13, 171-179.

Hunter, S.C., Cahoon, E.B., 2007. Enhancing vitamin E in oilseeds: unraveling tocopherol and tocotrienol biosynthesis. Lipids 42, 97-108.

Iinuma, M., Tosa, H., Tanaka, T., Riswan, S., 1996. Three new xanthones from the bark of Garcinia dioica. Chem. Pharm. Bull. 44, 232-234.

Jantan, I., Pisar, M.M., Idris, M.S., Taher, M., Ali, R.M., 2002. In vitro inhibitory effect of rubraxanthone isolated from Garcinia parvifolia on platelet-activating factor receptor binding. Planta Med. 68, 1133-1134.

Kapche, G.D.W.F., Fozing, C.D., Donfack, J.H., Fotso, G.W., Amadou, D., Tchana, A.N., Bezabih, M., Moundipa, P.F., Ngadjui, B.T., Abegaz, B.M., 2009. Prenylated arylbenzofuran derivatives from Morus mesozygia with antioxidant activity. 
Phytochemistry 70, 216-221.

Kim, J.H., Ryu, Y.B., Kang, N.S., Lee, B.W., Heo, J.S., Jeong, I.Y., Park, K.H., 2006. Glycosidase inhibitory flavonoids from Sophora flavescens. Biol. Pharm. Bull. 29, 302-305.

Kim, S.J., Son, K.H., Chang, H.W., Kang, S.S., Kim, H.P., 2003. Tyrosinase inhibitory prenylated flavonoids from Sophora flavescens. Biol. Pharm. Bull. 26, 1348-1350.

Kumano, T., Richard, S.B., Noel, J.P., Nishiyama, M., Kuzuyama, T., 2008. Chemoenzymatic syntheses of prenylated aromatic small molecules using Streptomyces prenyltransferases with relaxed substrate specificities. Bioorg. Med. Chem. 16, 8117-8126.

Kumazawa, S., Ueda, R., Hamasaka, T., Fukumoto, S., Fujimoto, T., Nakayama, T., 2007. Antioxidant prenylated flavonoids from propolis collected in Okinawa, Japan. J. Agri. Food Chem. 55, 7722-7725.

Kumazawa, S., Yoneda, M., Shibata, I., Kanaeda, J., Hamasaka, T., Nakayama, T., 2003. Direct evidence for the plant origin of Brazilian propolis by the observation of honeybee behavior and phytochemical analysis. Chem. Pharm. Bull. 51, 740-742.

Kuzuyama, T., Noel, J.P., Richard, S.B., 2005. Structural basis for the promiscuous biosynthetic prenylation of aromatic natural products. Nature 435, 983-987.

Laflamme, P., Khouri, H., Gulick, P., Ibrahim, R., 1993. Enzymatic prenylation of isoflavones in white lupin. Phytochemistry 34, 147-151.

Lee, J., Oh, W.K., Ahn, J.S., Kim, Y.H., Mbafor, J.T., Wandji, J., Fomum, Z.T., 2009. Prenylisoflavonoids from Erythrina senegalensis as novel HIV-1 protease inhibitors. Planta Med. 75, 268-270.

Lee, J.C., Kundu, J.K., Hwang, D.M., Na, H.K., Surh, Y.J., 2007. Humulone inhibits phorbol ester-induced COX-2 expression in mouse skin by blocking activation of NF-kappa B and AP-1: I kappa B kinase and c-Jun-N-terminal kinase as respective potential upstream targets. Carcinogenesis 28, 1491-1498.

Lee, M.H., Kim, J.Y., Ryu, J.H., 2005. Prenylflavones from Psoralea corylifolia inhibit nitric oxide synthase expression through the inhibition of I-אB- $\alpha$ degradation in activated microglial cells. Biol. Pharm. Bull. 28, 2253-2257.

Li, S.M., 2009. Evolution of aromatic prenyltransferases in the biosynthesis of indole derivatives. Phytochemistry, in press. 
Maeda, H., DellaPenna, D., 2007. Tocopherol functions in photosynthetic organisms. Curr. Opin. Plant Biol. 10, 260-265.

Mahmoud, Z.F., 1981. Antimicrobial components from Maclura pomifera fruit. Planta Med. 42, 299-301.

Mesia-Vela, S., Sanchez, R.I., Estrada-Muniz, E., Alavez-Solano, D., Torres-Sosa, C., Jimenez-Estrada, M., , Reyes-Chilpa, R., Kauffman, F.C., 2001. Natural products isolated from Mexican medicinal plants: novel inhibitors of sulfotransferases, SULT1A1 and SULT2A1. Phytomedicine 8, 481-488.

Miranda, C.L., Aponso, G.L.M., Stevens, J.F., Deinzer, M.L., Buhler, D.R., 2000. Prenylated chalcones and flavanones as inducers of quinone reductase in mouse Hepa 1c1c7 cells. Cancer Lett. 149, 21-29.

Oh, W.K., Kim, B.Y., Oh, H., Kim, B.S., Ahn, J.S., 2005. Phospholipase C $\gamma 1$ inhibitory activities of prenylated flavonoids isolated from Erythrina senegalensis. Planta Med. 71, 780-782.

Ohara, K., Muroya, A., Fukushima, N., Yazaki, K., 2009. Functional characterization of LePGT1, a membrane-bound prenyltransferase involved in the geranylation of p-hydroxybenzoic acid. Biochem. J. 421, 231-241.

Ohara, K., Yamamoto, K., Hamamoto, M., Sasaki, K., Yazaki, K., 2006. Functional characterization of OsPPT1, which encodes p-hydroxybenzoate polyprenyltransferase involved in ubiquinone biosynthesis in Oryza sativa. Plant Cell Physiol. 47, 581-590.

Okada, K., Ohara, K., Yazaki, K., Nozaki, K., Uchida, N., Kawamukai, M., Nojiri, H., Yamane, H., 2004. The AtPPT1 gene encoding 4-hydroxybenzoate polyprenyl diphosphate transferase in ubiquinone biosynthesis is required for embryo development in Arabidopsis thaliana. Plant Mol. Biol. 55, 567-577.

Moesta, P., Hahn, M.G., Grisebach, H., 1983. Development of a radioimmunoassay for the soybean phytoalexin glyceollin I. Plant Physiol. 73, 233-237.

Park, K.M., You, J.S., Lee, H.Y., Baek, N.T., Hwang, J.K., 2003. Kuwanon G: an antibacterial agent from the root bark of Morus alba against oral pathogens. J. Ethnopharmacol. 84, 181-185.

Pinto, M.M.M., Sousa, M.E., Nascimento, M.S.J., 2005. Xanthone derivatives: New insights in biological activities. Curr. Med. Chem. 12, 2517-2538.

Ribeiro, A.B., Abdelnur, P.V., Garcia, C.F., Belini, A., Severino, V.G., da Silva, M.F., 
Fernandes, J.B., Vieira, P.C., de Carvalho, S.A., de Souza, A.A., Machado, M.A., 2008. Chemical characterization of Citrus sinensis grafted on C. limonia and the effect of some isolated compounds on the growth of Xylella fastidiosa. J. Agric. Food Chem. 56, 7815-7822.

Robbins, M.P., Bolwell, G.P., Dixon, R.A., 1985. Metabolic changes in elicitor-treated bean cells. Eur. J. Biochem. 148, 563-569.

Row, E.C., Brown, S.A., Stachulski, A.V., Lennard, M.S., 2006. Synthesis of 8-geranyloxypsoralen analogues and their evaluation as inhibitors of CYP3A4. Bioorg. Med. Chem. 14, 3865-3871.

Sasaki, K., Tsurumaru, Y., Yazaki, K., 2009. Prenylation of flavonoids by biotransformation of yeast expressing plant membrane-bound prenyltransferase SfN8DT-1. Biosci. Biotechnol. Biochem. 73, 759-761.

Sasaki, K., Mito, K., Ohara, K., Yamamoto, H., Yazaki, K., 2008. Cloning and characterization of naringenin 8-prenyltransferase, a flavonoid-specific prenyltransferase of Sophora flavescens. Plant Physiol. 146, 1075-1084.

Shin, E. M., Zhou, H.Y., Guo, L.Y., Kim, J.A., Lee, S.H., Merfort, I., Kang, S.S., Kim, H.S., Kim, S., Kim, Y.S., 2008. Anti-inflammatory effects of glycyrol isolated from Glycyrrhiza uralensis in LPS-stimulated RAW264.7 macrophages. Int. Immunopharmacol. 8, 1524-1532.

Son, J.K., Park, J.S., Kim, J.A., Kim, Y., Chung, S.R., Lee, S.H., 2003. Prenylated flavonoids from the roots of Sophora flavescens with tyrosinase inhibitory activity. Planta Med. 69, 559-561.

Steffan, N., Grundmann, A., Yin, W.B., Kremer, A., Li, S.M., 2009. Indole prenyltransferases from fungi: a new enzyme group with high potential for the production of prenylated indole derivatives. Curr. Med. Chem. 16, 218-231.

Stevens, J.F., Page, J.E., 2004. Xanthohumol and related prenylflavonoids from hops and beer: to your good health! Phytochemistry 65, 1317-1330.

Stevens, J.F., Taylor, A.W., Nickerson, G.B., Ivancic, M., Henning, J., Haunold, A., Deinzer, M.L., 2000. Prenylflavonoid variation in Humulus lupulus: distribution and taxonomic significance of xanthogalenol and 4'-O-methylxanthohumol. Phytochemistry 53, 759-775.

Tielens, S., Wymeersch, F., Declercq, H., Cornelissen, M., 2008. Effect of 17 beta-estradiol on the in vitro differentiation of murine embryonic stem cells into 
the osteogenic lineage. In Vitro Cell. Dev. Biol. 44, 368-378.

Vitali, A., Giardina, B., Delle Monache, G., Rocca, F., Silvestrini, A., Tafi, A., Botta, B., 2004. Chalcone dimethylallyltransferase from Morus nigra cell cultures. Substrate specificity studies. FEBS Lett. 557, 33-38.

Welle, R., Grisebach, H., 1991. Properties and solubilization of the prenyltransferase of isoflavonoid phytoalexin biosynthesis in soybean. Phytochemistry 30, 479-484.

Wu, Q.L., Wang, S.P., Du, L.J., Yang, J.S., Xiao, P.G., 1998. Xanthones from Hypericum japonicum and H. Henryi. Phytochemistry 49, 1395-1402.

Yamahara, J., Kobayashi, G., Iwamoto, M., Chisaka, T., Fujimura, H., Takaishi, Y., Yoshida, M., Tomimatsu, T., Tamai, Y., 1990. Vasodilatory active principles of Sophora flavescens root. J. Ethnopharmacol. 29, 79-85.

Yamamoto, H., Senda, M., Inoue, K., 2000. Flavanone 8-dimethylallyltransferase in Sophora flavescens cell suspension cultures. Phytochemistry 54, 649-655.

Yazaki, K., Kunihisa, M., Fujisaki, T., Sato, F., 2002. Geranyl diphosphate: 4-hydroxybenzoate geranyltransferase from Lithospermum erythrorhizon. Cloning and characterization of a key enzyme in shikonin biosynthesis. J. Biol. Chem. 277, 6240-6246.

Zahringer, U., Ebel, J., Mulheirn, L.J., Lyne, R.L., Grisebach, H., 1979. Induction of phytoalexin synthesis in soybean. Dimethylallylpyrophosphate: trihydroxypterocarpan dimethylallyl transferase from elicitor-induced cotyledons. FEBS Lett. 101, 90-92.

Zuurbier, K.W.M., Fung, S.Y., Scheffer, J.J.C., Verpoorte, R., 1998. In-vitro prenylation of aromatic intermediates in the biosynthesis of bitter acids in Humulus lupulus. Phytochemistry 49, 2315-2322. 
Table 1

Polyphenol prenyltransferases involved in the biosynthesis of plant secondary metabolites.

\begin{tabular}{|c|c|c|c|}
\hline Species & Family & Prenylated compound & Prenyltransferase \\
\hline Sophora flavescens & Leguminosae & Sophoraflavanone G (1) & "Membrane-bound ${ }^{1)}$ \\
\hline Glycine max & Leguminosae & Glyceollin I (2) & Membrane-bound ${ }^{2)}$ \\
\hline Phaseolus vulgaris & Leguminosae & Phaseollin (3) & Membrane-bound $^{3)}$ \\
\hline Lupinus albus & Leguminosae & Wighteone (4) & Membrane-bound $^{4)}$ \\
\hline Morus nigra & Moraceae & Isocordoin (5) & Membrane protein $^{5}$ \\
\hline Humulus lupulus & Cannabaceae & $\begin{array}{l}\text { Xanthohumol (6) } \\
\text { Deoxyhumulone (7) }\end{array}$ & $\begin{array}{l}\text { Unidentified } \\
\text { Soluble }^{7)}\end{array}$ \\
\hline Cannabis sativa & Cannabaceae & $\begin{array}{l}\Delta^{1} \text {-Tetrahydrocannabinolic } \\
\text { acid (8) }\end{array}$ & Soluble $^{8)}$ \\
\hline $\begin{array}{l}\text { Macaranga } \\
\text { tanarius }\end{array}$ & Euphorbiaceae & Nymphaeol-A (9) & Unidentified $^{9)}$ \\
\hline Ammi majus & Umbelliferae & O-Prenylumbelliferone (10) & Membrane-bound $^{10)}$ \\
\hline $\begin{array}{l}\text { Hypericum } \\
\text { calycinum }\end{array}$ & Guttiferae & Hyperforin (11) & Soluble $^{11)}$ \\
\hline Ruta graveolens & Rutaceae & Demethylsuberosin (12) & Membrane-associated $^{12)}$ \\
\hline $\begin{array}{l}\text { Baccharis } \\
\text { dracunculifolia }\end{array}$ & Compositae & Artepillin C (13) & Unidentified $^{13)}$ \\
\hline
\end{tabular}

1) Yamamoto et al., 2000; 2) Welle and Grisebach, 1991; 3) Biggs et al., 1987; 4) Laflamme et al., 1993; 5) Vitali et al., 2004; 6) Stevens et al., 2000; 7) Zuurbier et al., 1998; 8) Fellermeier and Zenk, 1998; 9) Kumazawa et al., 2007; 10) Hamerski et al., 1990; 11) Boubakir et al., 2005; 12) Dhillon and Brown, 1976; 13) Kumazawa et al., 2003.<smiles>CC(C)CCC1CC(O)C(O)CC(O)C1C1CCC(O)CC1O</smiles>

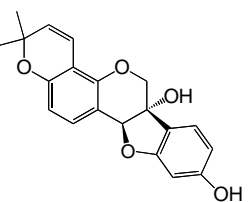

2

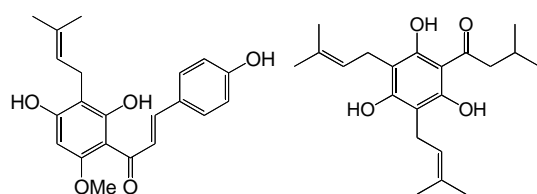
6 7

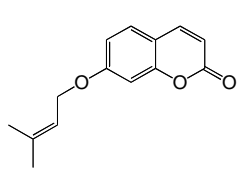

10

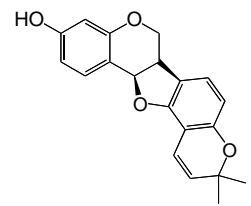

3

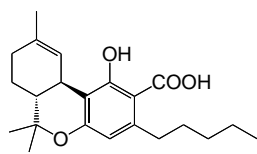<smiles>CC(C)=CCc1cc2ccc(=O)oc2cc1O</smiles><smiles>CC(C)CCC1C(O)CC2OCC(C3CCC(O)CC3)C(C)C2C1C</smiles><smiles>CC(C)CCC1C(O)CCC(CCC2CCCC2)C1O</smiles>

5<smiles>CC(C)=CCC/C(C)=C/CC1C(O)=CC2=C(C(=O)CC(c3ccc(O)c(O)c3)C2)C1O</smiles>

9<smiles>CC(C)=CCc1cc(/C=C/C(=O)O)cc(CC=C(C)C)c1O</smiles>

13 


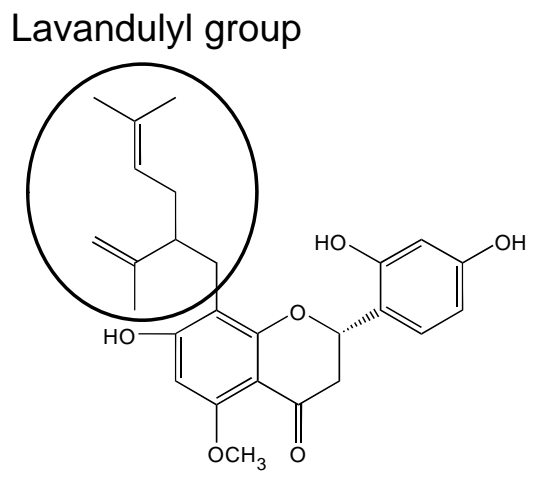

Kurarinone (Sophora flavescens)

$$
\text { Estrogen activity }
$$

Anti-tyrosinase

Anti-glycosidase

Inhibition of:

Cyclooxygenase (COX) Lipoxygenase (LOX) $\longrightarrow$ Prevent osteoporosis

$\longrightarrow$ Whitening effect

Prevention of diabetes Anti-infection

Anti-inflammatory

Anti-pain

Fig. 1. Types of biological activities of a prenylated flavonoid.

Kurarione is a flavanone with a lavandulyl group at the 8-position.

Fig. 1. Yazaki et al. 


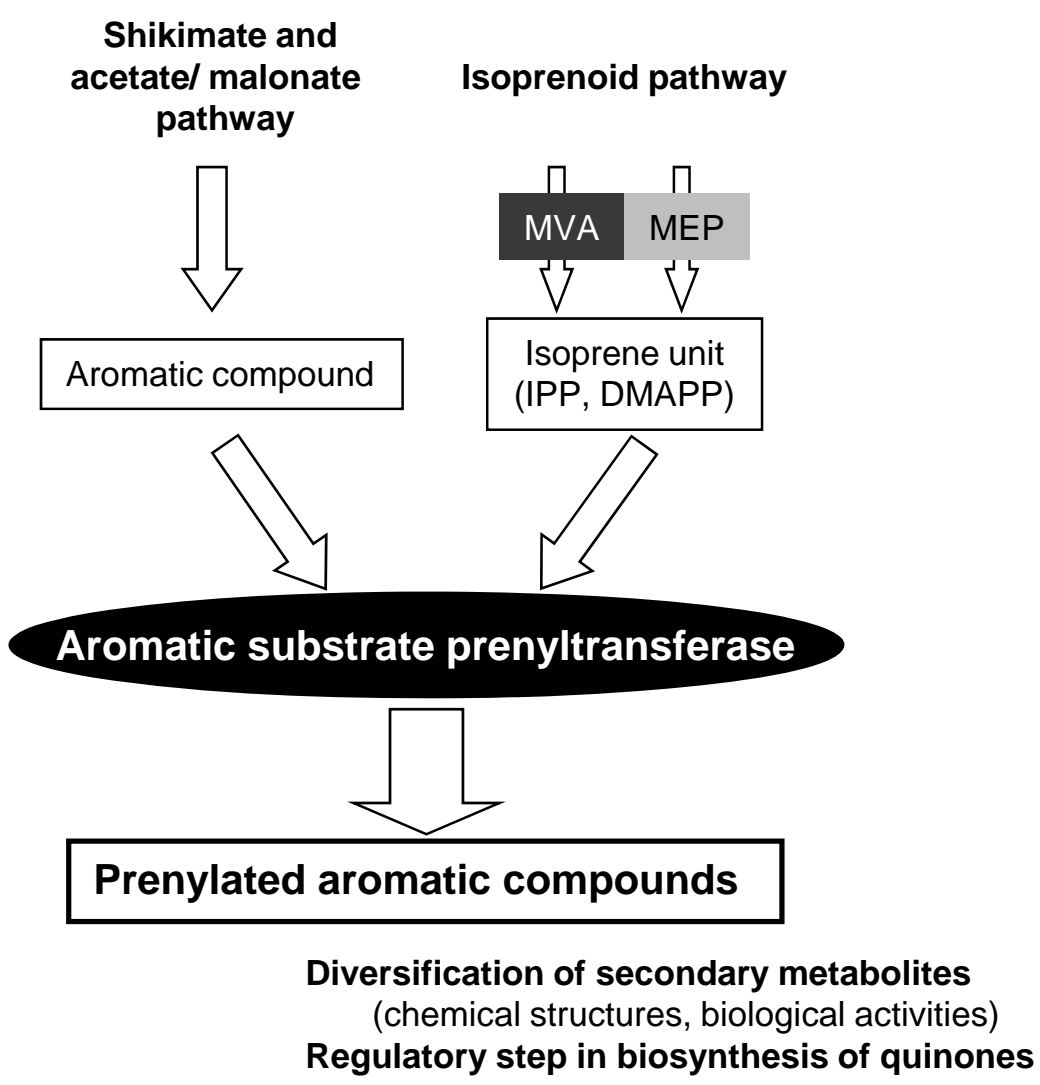

Fig. 2. Coupling reaction of the shikimate/ polyketide and isoprenoid pathways. The isoprenoid pathway consists of two pathways, the mevalonate (MVA) pathway localized in the cytosol, and the methyl erythritol phosphate (MEP) pathway localized in the plastid. Most plant prenyltransferases responsible for prenylated aromatic compounds are membrane-bound proteins. IPP, isopentenyl diphosphate.

Fig. 2. Yazaki et al. 


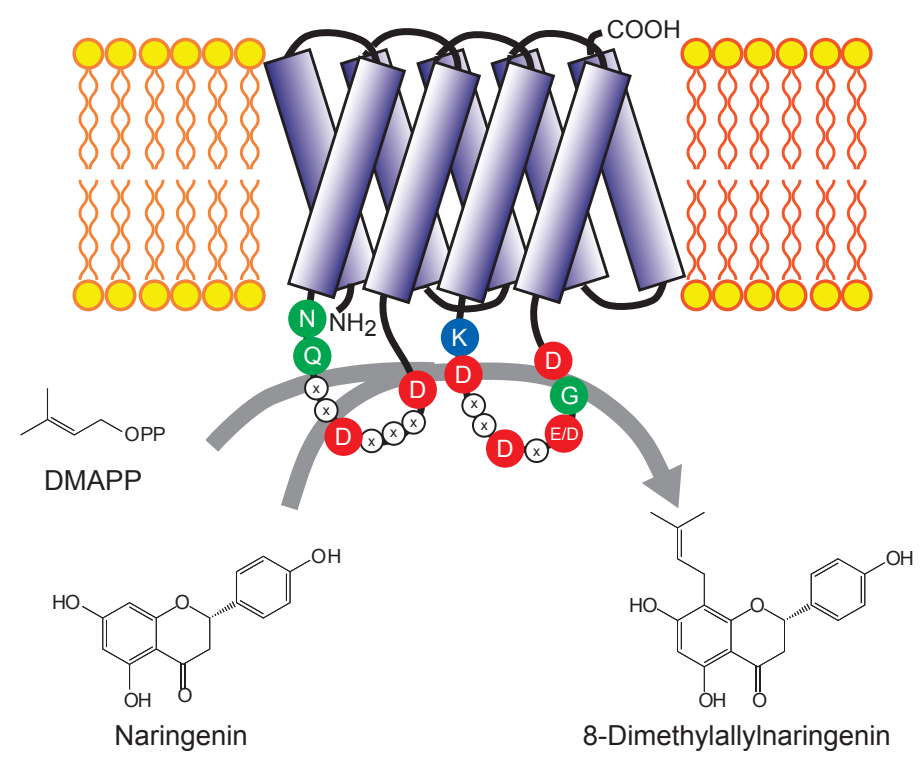

Fig. 3. The mechanism of the prenylation reaction and putative topology of the membrane-bound prenyltransferase SfN8DT-1.

SfN8DT-1 is presumed to have nine transmembrane a-helices and to be localized in the plastid. Flavonoid and homogentisate prenyltransferases possess two conserved aspartate-rich motifs, NQxxDxxxD and $\mathrm{KDxxDx}(\mathrm{E} / \mathrm{D}) \mathrm{GD}$, and prenyltransferases of $p$-hydroxy benzoate $(\mathrm{PHB})$ possess similar motifs. A recent study of PHB prenyltransferases suggested that these motifs are important for the prenylation reaction (Ohara et al., 2009).

Fig. 3. Yazaki et al. 


\section{HG geranylgeranyltransferase OsHGGT}

(End product: tocotrienol)

Plastid

HG phytyltransferase

Flavonoid dimethylallyltransferase

(End product: tocopherol)

(End product: prenylated flavonoid)

Plastid

Plastid

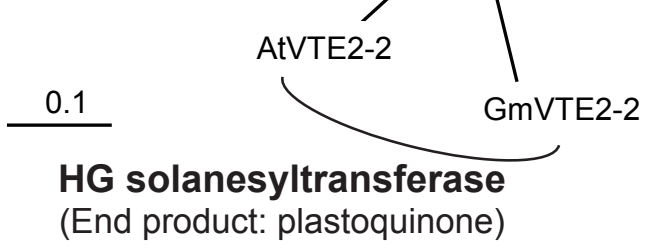

Plastid

Fig. 4. Phylogenetic relationship of prenyltransferases accepting aromatic substrates.

In this figure, PHB prenyltransferases are also included. A rooted phylogram was generated using a ClustalW alignment at GenomeNet (http://clustalw.genome.jp/).

Ap, Allium porrum; At, Arabidopsis thaliana; Cp, Cuphea pulcherrima; Gm, Glycine max; Hv, Hordeum vulgare; Le, Lithospermum erythrorhizon; Os, Oryza sativa; Ta, Triticum aestivum; Zm, Zea mays. Accession number: ApVTE2-1, DQ231057; AtHPT, AY089963; AtPPT1, AB052553; AtVTE2-2; DQ231060, CpHPT, DQ231058; GmG4DT, AB434690; GmHPT, DQ231059; GmVTE2-2, DQ231061; HvHGGT, AY222860; LePGT1, AB055078; LePGT2, AB055079; OsHGGT, AY222862; OsPPT1, AB263291; SfN8DT-1, AB325579; SfN8DT-2, AB370330; TaHGGT, AY222861; TaHPT; DQ231056, ZmHPT; DQ231055.

HG, homogentisate; PHB, $p$-hydroxybenzoate.

G4DT, glycinol 4-dimethylallyltransferase; HGGT, HG geranylgeranyltransferase; HPT, HG phytyltransferase; N8DT, naringenin 8-dimethylallyltransferase; PGT, PHB geranyltransferase; PPT, PHB prenyltransferase; VTE2-2, HG prenyltransferase.

Fig. 4. Yazaki et al. 